\title{
Las estrategias de profesionalización de las campañas presidenciales en Colombia desde 1994-2014*
}

\author{
Strategies to Professionalize the Presidential Campaigns in Colombia during 1994-2014 \\ Estratégias de profissionalização das campanhas presidenciais na Colômbia desde 1994-2014
}

\author{
Néstor Julián Restrepo-Echavarría ${ }^{a}$ \\ Universidad Eafit, Colombia \\ nrestr12@eafit.edu.co \\ ORCID: http://orcid.org/0000-0002-2881-1249 \\ Ángela María Gómez-Restrepo \\ Universidad de Medellin, Colombia \\ ORCID: http://orcid.org/0000-0002-2592-8097
}

DOI: https://doi.org/10.11144/Javeriana.syp38-74.epcp Redalyc: http://www.redalyc.org/articulo.oa $? \mathrm{id}=86059657004$

\section{Resumen:}

El propósito de este artículo es analizar una creciente modernización y adaptación del modelo norteamericano en las campañas presidenciales en Colombia desde 1994 hasta la campaña presidencial de 2014.

Todo proceso electoral tiene unas características particulares en las que se evidencia el impacto que tiene el sistema político de cada país sobre la organización interna y externa de las campañas electorales, las cuales se estructuran a partir del aprovechamiento de factores o técnicas de profesionalización, con el objetivo de lograr el mayor número de votantes.

Para entender este fenómeno se medirá el nivel de profesionalización de las campañas presidenciales en Colombia a partir de establecer unas categorías de análisis que permitan describir y comparar el desarrollo y evolución de las técnicas que incorpora cada campaña, lo que posibilitará establecer una comparación del nivel de profesionalización.

Palabras clave: Colombia, campañas electorales, profesionalización, nivel de profesionalización, candidatos, índice de profesionalización.

\section{Abstract:}

The purpose in this article is to analyze a growing modernization and adaptation of the American model to the presidential campaigns in Colombia from 1994 up to the campaigns in 2014. Any electoral process has some particular characteristics evincing the impact of the country's political system on both the internal and external organization of the electoral campaigns. These are structured based on the exploitation of professionalization techniques or factors aiming to gain the highest numbers of voters. To understand this phenomenon, the professionalization level of the presidential campaigns in Colombia will be measured by establishing some analysis categories allowing describing and comparing both the development and evolution of the techniques used in each campaign. All this would enable to compare the professionalization levels.

Keywords: Colombia, electoral campaigns, professionalization, professionalization level, candidates, professionalization index.

\section{Resumo:}

O objetivo deste artigo é analisar uma crescente modernização e adaptação do modelo norte-americano nas campanhas presidenciais na Colômbia desde 1994 até a campanha presidencial de 2014.

Todo processo eleitoral tem características particulares em que evidencia-se o impacto que tem o sistema político de cada país sobre a organização interna e externa das campanhas eleitorais, as quais estruturam-se a partir do aproveitamento de fatores ou técnicas de profissionalização, com o objetivo de alcançar o maior número de votantes.

Para entender esse fenómeno se mede o nível de profissionalização das campanhas presidenciais na Colômbia a partir de estabelecer categorias de análise que permitam descrever e comparar o desenvolvimento e evolução das técnicas incorporadas por cada campanha, o que possibilita estabelecer comparações do nível de profissionalização.

Palavras-chave: Colômbia, campanhas eleitorais, profissionalização, nível de profissionalização, candidatos, índice de profissionalização.

Notas de autor: 


\section{Introducción: definición y delimitación}

Las campañas electorales se han convertido en el escenario más destacado de la representación de la comunicación política. Su importancia radica en que es el momento de mayor relación entre los ciudadanos y la política y, por esta razón, intervienen -con sus respectivas estrategias discursivas y modos de interacciónlos candidatos, los partidos políticos que optan por la designación representativa, los medios de comunicación social y los ciudadanos.

Autores como Swanson y Mancini (1996), Norris (2001), Blumler (1999), Rospir (1999), Plasser y Plasser (2002), Maarek (2009), Kavanagh (2004) y Orejuela (2006) defienden la idea de que el fenómeno de la profesionalización de las campañas electorales tiene su origen en las norteamericanas, las cuales se caracterizan por su alta competencia y, también, por su innovación al centrarlas en los medios de comunicación.

De igual manera, la profesionalización de las campañas se puede enmarcar en todos los cambios y transformaciones políticas del siglo XX, principalmente como resultado de la aparición de las nuevas tecnologías de la información y su efecto sobre el debate, el discurso político y las diferentes estrategias de búsqueda del poder (Castells, 2009).

Crespo, Garrido y Riorda (2008) señalan que las causas de la aparición de este fenómeno en la política pueden atribuirse a los siguientes factores: el declive de los partidos políticos y el de su papel a la hora de seleccionar los candidatos; el surgimiento de un sistema de medios de comunicación complejo, centrado en la televisión pero con una diversidad creciente de medios flexibles e interconectados electrónicamente; y el desarrollo del marketing político, en especial la aparición de las encuestas de opinión, la segmentación de públicos y la utilización de todo tipo de herramientas de tele mercadeo -correo informatizado y bases de datos telefónicos- (Restrepo-Echavarría, 2017).

En efecto, durante el periodo en que se realizan las campañas electorales, los candidatos o partidos ofrecen información a los ciudadanos respecto a los programas de gobierno y las propuestas que se pretenden ejecutar en caso de ser elegidos. Por su parte, los ciudadanos están interesados en escuchar las propuestas y las diversas noticias del momento electoral, para lo cual los medios de comunicación asumen un rol fundamental, ya que son estos los encargados de la difusión (Restrepo-Echavarría, 2017).

Las estrategias que se diseñan en el proceso electoral, relacionadas con la información, publicidad, propaganda e interacción comunicativa, han interesado a los investigadores de las ciencias sociales y a los expertos en el desarrollo práctico del marketing político. Sin embargo, la constante adaptación e innovación de dichos mecanismos y procesos obliga a una permanente actualización de la investigación y de los conocimientos derivados de ella, por lo que se requiere comprender, en su justa medida, la repercusión que la comunicación política puede tener en la evolución de los instrumentos y estrategias comunicativas en la vida política, y su potencial incidencia en la evolución de la democracia.

Por lo anterior, en este artículo se analizan las campañas presidenciales en Colombia desde 1994-momento en el que se generan cambios sustanciales en el entorno político nacional e internacional que conllevan una nueva constitución política, la cual modifica el diseño institucional del Estado- hasta 2014, última campaña electoral en la que se evidencia una transformación en la forma de hacer las campañas presidenciales al tratar de identificar el proceso de profesionalización y evolución en la forma de hacer las compañas políticas.

\section{Descripción de las campañas electorales en Colombia}

Colombia se ha caracterizado por tener una tradición electoral fundamentada en la continuidad de sus procesos electorales. Las campañas electorales en Colombia se desarrollaron dentro de la caracterización de las campañas premodernas, en las que la comunicación política estuvo basada en la influencia de los partidos 
políticos tradicionales, la ideología partidista, el contacto cara a cara, y en las que los ciudadanos basaban su elección en la disciplina y la lealtad hacia el partido de acervo y tradición.

En efecto, durante esta época Restrepo-Echavarría (2017 plantea que la estructura de la organización de las campañas dependía de los dirigentes, quienes se encargaban de la elaboración de spots de radio como "Liberal vota por Liberal", de medios impresos como "Liberal vota por tu jefe Liberal", y realizaban correrías que buscaban la favorabilidad de los seguidores del partido mediante el patronazgo y el clientelismo (RestrepoEchavarría, 2017.

Es difícil indagar sobre estudios que construyan los inicios de la comunicación política en Colombia, ya que la costumbre electoral se basa en la competencia de los partidos políticos tradicionales, con su institucionalidad para realizar la estrategia propagandística, y la lucha ideológica de ambas facciones y alta militancia. Sin embargo, estudios como el de Juan Lozano y Lozano, Jardín de Candidato: una campaña presidencial, y el de José Restrepo Restrepo y Belisario Betancur, Historia de una campaña política, son textos que hicieron evidente la modernización de los procesos electorales en el país a partir de nuevos antecedentes y técnicas, en las que el partido político le abre paso a la personalización de la política, el uso de los medios de comunicación y la señalética propagandística con bajos niveles de propaganda partidista, y el aumento exponencial del uso de los medios de comunicación - prensa, radio y televisión-, a favor o en contra de los liderazgos políticos. De igual manera, permitieron analizar diferentes alianzas interpartidistas impensables en un bipartidismo dogmático y cerrado como el que hubo durante gran parte del siglo XX.

Luego, en la década de 1990, la crisis institucional y partidista generada por la corrupción, el conflicto armado, la falta de legitimidad de las instituciones del Estado y el estancamiento de los partidos políticos tradicionales, conllevó la promulgación de la Constitución Política de 1991, que modificó el diseño institucional en los siguientes términos: en el sistema de partidos se pasó de un sistema bipartidista a uno multipartidista, en el que toda la población tiene representación política; y en el sistema electoral se establecieron las reglas que permitirían la segunda vuelta para la elección presidencial, lo que legitima el poder del ganador. En adición, se introdujeron cambios en el esquema de financiación de las campañas, al tomar vigencia el sistema de financiación mixta; se potenciaron las campañas subnacionales a partir de la elección de alcaldes y gobernadores; y, por último, se implementó el tarjetón electoral en el que la imagen del candidato prima sobre el logo del partido.

A partir de esta época se empieza a evidenciar la modernización de las campañas electorales a nivel nacional y subnacional, sin dejar de lado las tradiciones electorales propias de la cultura colombiana.

Estudios como los de Becassino (2008 y Restrepo-Echavarría (2015, 2018 describen el papel de los medios de comunicación en las campañas electorales, el uso de la comunicación política y las técnicas de marketing político, y el manejo de las campañas electorales, no sólo desde una dimensión táctica, sino también estratégica, lo que permite abordar dos áreas: la campaña de imagen y la electoral, en las que el foco se centra en el candidato como persona que tiene proximidad con el elector, y se dejan de lado las lealtades partidistas e ideológicas.

En las últimas campañas electorales en Colombia se ha evidenciado la creciente importancia y el desarrollo acelerado de la asesoría política, de los asesores en publicidad, y de las nuevas tecnologías en el ámbito nacional, como lo demuestran innumerables firmas de asesoría, como Dattis, Germán Medina \& Asociados y Global News Intelligence Latinoamérica.

Así mismo, hay un gran número de asesores foráneos que también hacen campañas electorales en Colombia: Juan José Rendón, los mexicanos Roberto Trad y Roy Campos, el argentino Felipe Noguera, el director de la maestría de Comunicación de Gobierno de la Universidad George Washington (EE. UU, Roberto Izurrieta, los brasileños Carlos Manhanelly y Duda Mendioca, el chileno Hernán Larrain, los mexicanos Juan Carlos Limón, David Ross y Sergio Torres Ávila, y los españoles Juan Marcos Magaña Rodríguez y Yago de Marta (Restrepo-Echavarría, 2017. 
Becassino (2008) argumenta que todo lo anterior ha dado lugar a un incremento de campañas electorales que explotan la cultura del consumo por impulso, y cuyo énfasis está puesto en la agenda, el discurso y la imagen, al emplear los sentimientos públicos coyunturales sin preocuparse por construir relaciones permanentes con los electores.

De la misma forma advierte que, en la mayoría de países de América Latina, las campañas electorales se centran en los candidatos que buscan marcar los puntos negativos de sus adversarios, las encuestas de opinión y la escenificación de la coyuntura, sin proponer soluciones estructurales para los ciudadanos.

En suma, se puede argumentar que las campañas presidenciales en Colombia han migrado paulatinamente de las calles y plazas públicas a los debates de televisión, a las correrías políticas, a los foros virtuales, y a salas de chat y redes sociales; y están más enfocadas en la seguridad y el control nacional, que en otros aspectos como el desempleo, la corrupción y la economía.

\section{Metodología: fórmula para la medición del nivel de profesionalización de las campañas presidenciales en Colombia}

Todo proceso electoral tiene unas características particulares en las que se evidencia el impacto que tiene el sistema político de cada país sobre la organización interna y externa de las campañas electorales. En una campaña profesional se log ran evidenciar dos dimensiones: la dimensión organizacional, que comprende los aspectos relacionados con la estructuración interna de la campaña electoral, la cual es entendida como una empresa electoral con una organización y unas áreas funcionales que son estratégicas, tácticas y técnicas (Restrepo-Echavarría, 2017); y la dimensión comunicativa, cuya pretensión es la de obtener mayor efectividad de las campañas. Para los profesionales del marketing político es fundamental la adopción de ciertos recursos comunicacionales como camino hacia el elector, quien está cada vez menos identificado con partidos o agrupamientos sociales. De esta manera, los mensajes de las campañas se orientan hacia las siguientes características: el foco en la imagen, que hace del político la traducción misma de las expectativas del elector; la superficialidad ajustada al sentimiento de un ciudadano poco atento a las discusiones profundas; y el enfoque simbólico, que tiene una intención general o recursos visuales sofisticados (Rocha, 2007). 
TABLA 1

Dimensión organizacional y dimensión comunicativa de campañas presidenciales en Colombia

\begin{tabular}{|c|c|}
\hline Categoría & Indicadores dimensión organizacional \\
\hline \multirow[t]{6}{*}{ Estratégica } & Comando de las Campañas: Centralizado vs. Descentralizado. \\
\hline & Presencia de consultores externos en áreas de comando. \\
\hline & $\begin{array}{l}\text { Fuentes de financiación autónoma, con técnicas para recaudar fondos y ajena a la estructura regular de los } \\
\text { partidos. }\end{array}$ \\
\hline & $\begin{array}{l}\text { Uso de investigación social para el seguimiento de las percepciones y reacciones del electorado (manejo de } \\
\text { la agenda pública y coyuntura política) }\end{array}$ \\
\hline & $\begin{array}{l}\text { Campaña permanente. Rompiendo el acontecimiento rígido y estrecho de un marco temporal para la } \\
\text { comunicación de campańa. }\end{array}$ \\
\hline & Gerenciamiento profesional de campañas. \\
\hline \multirow[t]{5}{*}{ Táctica } & Sistematización de banco de datos. \\
\hline & La profesionalización del grupo de campaña, un equipo de trabajo multi e interdisciplinario. \\
\hline & Militancia remunerada en la campańa. \\
\hline & $\begin{array}{l}\text { Personalización de la campaña en el candidato alejándose de los de programas y orientaciones ideológicas } \\
\text { del partido (Personalización de la política- Outsider) }\end{array}$ \\
\hline & Convenciones y Alianzas interpartidistas. Apoyos (Multi- avales) \\
\hline \multirow[t]{2}{*}{ Técnica } & Monitoreo Electoral: Uso de sondeos, encuestas y grupos focales \\
\hline & Media Training y entrenamiento de equipo \\
\hline \multirow[t]{5}{*}{ Discursiva } & Mensajes Personalizados \\
\hline & Uso de referentes simbólico \\
\hline & Segmentación de los mensajes \\
\hline & Uso emocional en los mensajes electorales \\
\hline & Nuevas formas de discurso político \\
\hline \multirow{4}{*}{$\begin{array}{l}\text { Procesos de } \\
\text { Comunicación }\end{array}$} & Construcción de imagen y posicionamiento del candidato \\
\hline & Storytelling (historias) \\
\hline & Participación en debates televisados y en foros sociales. \\
\hline & Uso de propaganda negativa \\
\hline \multirow{5}{*}{$\begin{array}{l}\text { Medios } \\
\text { Técnicos }\end{array}$} & Inserciones publicitarias en medios masivos de comunicación: Radio y televisión pública y privada \\
\hline & Uso de Internet \\
\hline & $\begin{array}{l}\text { Utilización de material escrito para uso externo en las campañas electorales: Prensa no partidista, material } \\
\text { publicitario, vallas públicas, Posters, afiches, material POP' }\end{array}$ \\
\hline & Uso de e-mail y correos personalizados para contactos con los votantes. \\
\hline & Uso de nuevos recursos: Desde SMS hasta WhatsApp \\
\hline
\end{tabular}

Fuente: elaboración propia a partir de Restrepo-Echavarría, Rodríguez-Díaz y Castromil (2018), Norris (2004), Plasser y Plasser (2002), Gibson y Römmele (2001, 2009), Rocha (2007), Mancini (1995), Maarek (2009), Swanson y Mancini (1996), Rospir (1999) y Kavanagh (2004)

${ }^{*}$ El material POP (por sus siglas en inglés: Point-of-Purchase) es el material promocional colocado en las tiendas para captar la atención del consumidor e impulsarlo a comprar. Incluye los letreros que se colocan en los estantes, anuncios en las ventanas, módulos de demostración, entre otros. 
Para medir el nivel de profesionalización de las campañas presidenciales en Colombia, considerando las dimensiones organizacional y comunicativa explicadas, se debe tener en cuenta el nivel de intensidad de cada uno de los indicadores que componen cada dimensión, los cuales se detallan en la tabla anterior. La intensidad representa la ocurrencia de cada uno de los indicadores de profesionalización en cada una de las campañas presidenciales. De este modo, cuando el indicador se presenta con alta frecuencia se calificará con una intensidad de dos (2), cuando sea de mediana frecuencia se calificará con una intensidad de uno (1) y si el indicador se presenta con baja frecuencia, o incluso es inexistente, se calificará con una intensidad de cero (0). De este modo, las campañas serán más profesionales en la medida que sus indicadores de profesionalización se presenten con alta frecuencia y, por tanto, tengan la mayor calificación de intensidad.

TABLA 2

Intensidad de los indicadores de profesionalización de las campañas presidenciales en Colombia

\begin{tabular}{|c|c|c|c|c|}
\hline \multirow{2}{*}{ Categoría } & \multirow{2}{*}{ Indicador } & \multicolumn{3}{|c|}{ Intensidad } \\
\hline & & 0 & 1 & 2 \\
\hline \multicolumn{5}{|c|}{ Dimensión Organizativa } \\
\hline \multirow{6}{*}{ 莺 } & $\begin{array}{l}\text { Comando de las Campañas: Centralizado } \\
\text { vs. Descentralizado. }\end{array}$ & Descentralizado & Parcial & Total \\
\hline & $\begin{array}{l}\text { Presencia de consultores externos en áreas } \\
\text { de comando. }\end{array}$ & $\begin{array}{l}\text { Poco o nada } \\
\text { representativo }\end{array}$ & $\begin{array}{l}\text { Participaci } \\
\text { ón } \\
\text { mediana } \\
\end{array}$ & $\begin{array}{c}\text { Participación } \\
\text { con alta } \\
\text { importancia }\end{array}$ \\
\hline & Gestión de la Financiación & Bajo & Medio & Alto \\
\hline & $\begin{array}{l}\text { Uso de investigación social para el } \\
\text { seguimiento de las percepciones y } \\
\text { reacciones del electorado }\end{array}$ & Baja & Media & Alta \\
\hline & Campaña permanente. & Bajo & Mediana & Alta \\
\hline & Gerenciamiento profesional de campañas. & $\begin{array}{l}\text { Esporádico / } \\
\text { Inexistente }\end{array}$ & Frecuente & $\begin{array}{c}\text { Muy } \\
\text { frecuente }\end{array}$ \\
\hline \multirow{5}{*}{ 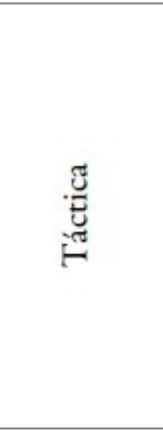 } & Sistematización de banco de datos. & $\begin{array}{c}\text { Bajo / } \\
\text { Inexistente }\end{array}$ & $\begin{array}{c}\text { Uso } \\
\text { mediano }\end{array}$ & Amplio uso \\
\hline & $\begin{array}{l}\text { La profesionalización del grupo de } \\
\text { campaña. }\end{array}$ & $\begin{array}{c}\text { Bajo / } \\
\text { Inexistente }\end{array}$ & Mediano & Alto \\
\hline & Militancia remunerada en la campaña. & Voluntaria & Mezclada & Pagada \\
\hline & $\begin{array}{l}\text { Personalización de la campaña en el } \\
\text { candidato. }\end{array}$ & Baja & Media & Alta \\
\hline & $\begin{array}{l}\text { Convenciones y Alianzas interpartidistas. } \\
\text { Apoyos }\end{array}$ & $\begin{array}{c}\text { Bajo / } \\
\text { Inexistente }\end{array}$ & Medio & Alta \\
\hline \multirow{2}{*}{ 苞 } & $\begin{array}{l}\text { Monitoreo Electoral: Uso de sondeos, } \\
\text { encuestas y grupos focales }\end{array}$ & $\begin{array}{c}\text { Bajo / } \\
\text { Inexistente }\end{array}$ & Medio & Alto \\
\hline & Media Training y entrenamiento de equipo & $\begin{array}{c}\text { Bajo / } \\
\text { Inexistente }\end{array}$ & Mediano & Alto \\
\hline
\end{tabular}

Fuente: elaboración propia a partir de Restrepo-Echavarría et al. (2018), Norris (2004), 
TABLA 2 (CONT.)

Intensidad de los indicadores de profesionalización de las campañas presidenciales en Colombia

\begin{tabular}{|c|c|c|c|c|}
\hline \multirow{2}{*}{ Categoría } & \multirow{2}{*}{ Indicador } & \multicolumn{3}{|c|}{ Intensidad } \\
\hline & & 0 & 1 & 2 \\
\hline \multicolumn{5}{|c|}{ Dimensión Comunicativa } \\
\hline \multirow{5}{*}{ 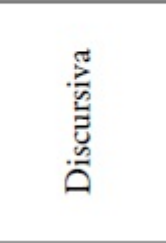 } & Mensajes Personalizados & Bajo & Mediano & Alta \\
\hline & Uso de referentes simbólico & Bajo & Mediano & Alta \\
\hline & Segmentación de los mensajes & Bajo & Mediano & Alta \\
\hline & Uso emocional en los mensajes electorales & Bajo & Mediano & Alta \\
\hline & Nuevas formas de discurso político & Bajo & Mediano & Alta \\
\hline \multirow{4}{*}{ 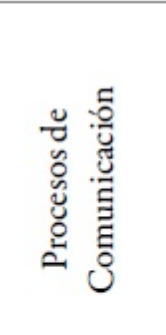 } & $\begin{array}{l}\text { Construcción de imagen y posicionamiento } \\
\text { del candidato }\end{array}$ & Bajo & Mediano & Alta \\
\hline & Storytelling (historias) & Bajo & Mediano & Alta \\
\hline & $\begin{array}{l}\text { Participación en debates televisados y en } \\
\text { foros sociales. }\end{array}$ & Bajo & Mediano & Alta \\
\hline & Uso de propaganda negativa & Bajo & Mediano & Alta \\
\hline \multirow{5}{*}{ 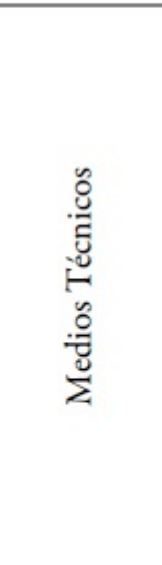 } & $\begin{array}{l}\text { Inserciones publicitarias en medios masivos } \\
\text { de comunicación }\end{array}$ & $\begin{array}{c}\text { Esporádico / } \\
\text { Inexistente }\end{array}$ & Frecuente & $\begin{array}{c}\text { Muy } \\
\text { frecuente }\end{array}$ \\
\hline & Uso de Internet & $\begin{array}{c}\text { Esporádico / } \\
\text { Inexistente }\end{array}$ & Frecuente & $\begin{array}{c}\text { Muy } \\
\text { frecuente }\end{array}$ \\
\hline & $\begin{array}{l}\text { Utilización de material escrito para uso } \\
\text { externo en las campañas electorales }\end{array}$ & $\begin{array}{c}\text { Esporádico / } \\
\text { Inexistente }\end{array}$ & Frecuente & $\begin{array}{c}\text { Muy } \\
\text { frecuente }\end{array}$ \\
\hline & $\begin{array}{l}\text { Uso de email y correos personalizados para } \\
\text { contactos con los votantes }\end{array}$ & $\begin{array}{c}\text { Esporádico / } \\
\text { Inexistente }\end{array}$ & Frecuente & $\begin{array}{c}\text { Muy } \\
\text { frecuente }\end{array}$ \\
\hline & $\begin{array}{l}\text { Uso de nuevos recursos: Desde SMS hasta } \\
\text { WhatsApp }\end{array}$ & $\begin{array}{c}\text { Esporádico / } \\
\text { Inexistente }\end{array}$ & Frecuente & $\begin{array}{c}\text { Muy } \\
\text { frecuente }\end{array}$ \\
\hline
\end{tabular}

Fuente: elaboración propia a partir de Restrepo-Echavarría et al. (2018), Norris (2004),

Plasser y Plasser (2002), Gibson y Römmele (2001, 2009), Rocha (2007), Mancini

(1995), Maarek (2009), Swanson y Mancini (1996), Rospir (1999) y Kavanagh (2004)

El índice de profesionalización total $\left(\right.$ INPROCO $\left._{T}\right)$ se construirá a partir del promedio de la intensidad promedio de profesionalización de cada una de las dimensiones. Con esto, se pretende comprender cuál dimensión es la que permite explicar el nivel de profesionalización de las campañas presidenciales en Colombia. La intensidad de profesionalización promedio de cada una de las dimensiones será un valor que oscila entre cero (0) y dos (2), y las fórmulas que permiten su cálculo se indican en la fórmula 1 y la fórmula 2 , y en la fórmula 3 se establece el cálculo del índice de profesionalización total $\left(I_{N P R O C O}\right)$ (RestrepoEchavarría et al., 2018).

$$
I N P R O C O_{o}=\frac{\sum_{i=1}^{13} \text { Intensidad Indicadores Dimensión Organizativa }_{i}}{13}
$$

Donde: INPROCOO representa la intensidad promedio de profesionalización de la Dimensión Organizativa. 


$$
\text { INPROCO }_{C}=\frac{\sum_{i=1}^{14} \text { Intensidad Indicadores Dimensión Comunicativa }_{i}}{14}
$$

Donde: $\operatorname{INPROCO}_{O}$ representa la intensidad promedio de profesionalización de la Dimensión Organizativa.

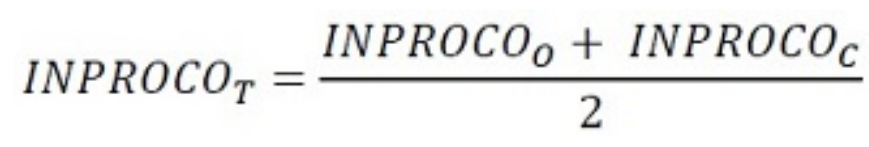

Donde: INPROCO $O$ representa la intensidad promedio de profesionalización de la Dimensión Organizativa e INPROCO ${ }_{C}$ representa la intensidad promedio de profesionalización de la Dimensión Comunicativa.

El índice de profesionalización total $\left(I N P R O C O_{T}\right)$ es un valor que oscilará entre cero (0) y dos (2), y los niveles de profesionalización de las campañas se detallan en la tabla 3. Cabe anotar que bajo esta misma escala se pueden analizar los niveles de profesionalización de la dimensión organizativa y comunicativa (RestrepoEchavarría et al., 2018).

TABLA 3

Niveles de profesionalización de las campañas electorales según el índice INPROCO

\begin{tabular}{ccc}
\hline $\begin{array}{c}\text { Índice de Profesionalización } \\
(\text { INPROCO) }\end{array}$ & $\begin{array}{c}\text { Nivel de } \\
\text { Profesionalización }\end{array}$ & $\begin{array}{c}\text { Porcentaje de } \\
\text { Profesionalización }\end{array}$ \\
\hline $0 \leq$ INPROCO $_{T}<0,5$ & Bajo & $0 \% \leq$ INPROCO $_{T}<25 \%$ \\
\hline $0,5 \leq$ INPROCO $_{T}<1$ & Medio & $25 \% \leq$ INPROCO $_{T}<50 \%$ \\
\hline $1 \leq$ INPROCO $_{T}<1,5$ & Medio -Alto & $50 \% \leq$ INPROCO $_{T}<75 \%$ \\
\hline $1,5 \leq$ INPROCO $_{T} \leq 2$ & Alto & $75 \% \leq$ INPROCO $_{T} \leq 100 \%$ \\
\hline
\end{tabular}

Fuente: elaboración propia a partir de Rocha (2007) y Restrepo-Echavarría et al. (2018)

\section{Resultados}

El resultado de este análisis es la cuantificación del índice INPROCOt, que es el que permitirá medir el nivel de profesionalización de cada una de las campañas presidenciales en Colombia desarrolladas desde la década de 1990 hasta la campaña presidencial de 2014. Cabe anotar que se estudiaron las campañas de los candidatos que obtuvieron el mayor número de votos en cada una de las contiendas electorales, los cuales se indican en la tabla. 
TABLA 4

Campañas presidenciales en Colombia desde 1990 hasta 2014

\begin{tabular}{ccc}
\hline $\begin{array}{c}\text { Periodo de } \\
\text { Análisis }\end{array}$ & $\begin{array}{c}\text { Periodo } \\
\text { Presidencial }\end{array}$ & \multicolumn{1}{c}{ Candidatos } \\
\hline Década de 1990 & $1994-1998$ & Ernesto Samper Pizano - Andrés Pastrana Arango \\
\cline { 2 - 3 } & $1998-2002$ & Andrés Pastrana Arango - Horacio Serpa \\
\hline Década de 2000 & $2002-2006$ & Álvaro Uribe Vélez - Horacio Serpa Uribe \\
\cline { 2 - 3 } & $2006-2010$ & Álvaro Uribe Vélez - Carlos Gaviria Díaz \\
\hline Década de 2010 & $2010-2014$ & Juan Manuel Santos - Antanas Mockus Sivikas \\
\cline { 2 - 3 } & $2014-2016$ & Juan Manuel Santos - Oscar Iván Zuluaga \\
\hline
\end{tabular}

Fuente: elaboración propia a partir de información de las campañas presidenciales

\section{Medición y análisis del nivel de profesionalización total: campaña 1994}

Para 1994, la campaña electoral se desplegó en el marco de la disputa bipartidista entre el Partido Conservador y el Partido Liberal, que eran los de tradición electoral en el país y los que habían ostentado el poder político por décadas en las que se había presentado una alternancia del poder y se había mantenido una vigencia electoral equiparable. En esta contienda electoral, los candidatos Andrés Pastrana Arango y Ernesto Samper Pizano se enfrentaron por la Presidencia de la República, y al medir el nivel de profesionalización de ambas campañas se logró evidenciar un nivel medio de profesionalización $\left(0,5 \leq I N P R O C O_{T}<1\right)$, en el cual la campaña de Andrés Pastrana obtuvo un nivel más alto de profesionalización que la de Ernesto Samper. Así mismo, se encontró que el nivel de profesionalización de las campañas en este periodo se debe a la dimensión comunicativa más que a la organizativa. En promedio, el nivel de profesionalización de la dimensión comunicativa es de 1,04, y de la organizativa es de 0,69 $\left(\right.$ INPROCO $_{C}>$ INPROCO $\left._{O}\right)$.

A partir de los resultados se puede concluir que, durante esta contienda electoral, los partidos políticos tradicionales tenían una alta influencia sobre la estructura de las campañas electorales, y que éstas se desarrollaron bajo las lógicas de las usanzas tradicionales, sin altos niveles de profesionalización y en las cuales las herramientas de comunicación tradicionales fueron clave para la construcción de un mensaje emocional para los electores. Además, el mayor nivel de profesionalización en la dimensión comunicativa se explica por la necesidad que tuvieron los candidatos de pasar de la plaza pública al debate televisado como consecuencia de la violencia que había impactado la contienda de 1990.

\section{Medición del nivel de profesionalización Dimensión Comunicativa: campaña 1998}

En el proceso electoral de 1998 se evidencia que ambas campañas tuvieron un nivel medio- alto de profesionalización en la dimensión comunicativa $\left(0,5 \leq I N P R O C O_{C}<1,5\right)$, y que la campaña de Andrés Pastrana obtuvo un nivel más alto de profesionalización en esta dimensión que la de Horacio Serpa. En la categoría discursiva se evidencia que la campaña de Pastrana se diferencia de la de Serpa en la incorporación de nuevas formas de discurso político basadas en el mensaje de renovación, alejándose así de las instituciones partidistas tradicionales y dando inicio a los mensajes apolíticos sin bases ideológicas.

En particular, el nivel de profesionalización de esta categoría es alto para la campaña de Pastrana (1,5 $\leq$ INPROCO $_{C}$ : Categoría Dicursiva $\left.<2\right)$ y medio-alto para la de Serpa $\left(1,0 \leq\right.$ INPROCO $_{C}$ : Categoría 
Dicursiva $<1,5)$. En cuanto a la categoría de procesos comunicacionales, ambas campañas tuvieron un nivel de profesionalización medio-alto $\left(1,0 \leq I N P R O C O_{C}\right.$ : Categoría Procesos Comunicacionales < 1,5); sin embargo, en esta categoría la campaña de Pastrana tuvo un nivel de profesionalización superior a la de Serpa, lo cual se explica por la alta personalización de la campaña en la imagen del candidato. Finalmente, en la categoría de medios técnicos se evidencia un nivel de profesionalización medio de ambas campañas $\left(0,5 \leq I N P R O C O_{C}\right.$ : Categoría Medios Técnicos < 1,0), que es el resultado del uso intensivo de medios de comunicación tradicionales y del material publicitario.

\section{Medición y análisis del nivel de profesionalización total: campaña 1998}

La campaña de 1998 planteó muchos cambios al panorama político, los cuales se ven directamente relacionados con la implementación de nuevas formas de desarrollar las campañas, con más profesionalismo y organización en todas sus dimensiones. En estas elecciones, el candidato Andrés Pastrana Arango tuvo un nivel de profesionalización medio-alto $\left(1 \leq I N P R O C O_{T}<1,5\right)$, mientras que Horacio Serpa tuvo un nivel medio $\left(0,5 \leq \operatorname{INPROCO}_{T}<1\right)$. Así mismo, se encontró que el nivel de profesionalización de las campañas en este periodo se debe a la dimensión comunicativa más que a la organizativa; en promedio, el nivel de profesionalización de la dimensión comunicativa es de 1,21, y el de la organizativa es de 0,96 (INPROCO $>$ INPROCOO ).

\section{Medición del nivel de profesionalización dimensión comunicativa: campaña 2002}

En el proceso electoral de 2002 se evidencia que ambas campañas tuvieron un nivel medio-alto de profesionalización en la dimensión comunicativa $\left(1 \leq I N P R O C O_{C}<1,5\right)$, con un 1,29 . Tanto la categoría discursiva, como la de procesos de comunicación y la de medios técnicos, tienen un nivel medio-alto de profesionalización ${ }^{1}$ y la misma calificación para ambas campañas, lo que evidencia que los efectos comunicativos se fueron estandarizando en las campañas colombianas.

De manera particular, y en comparación con las campañas de la década de 1990, se encontró una evolución en el uso intensivo de herramientas discursivas, específicamente en la segmentación de los mensajes y en el uso de medios técnicos, especialmente el Internet y el correo electrónico, que no habían sido incorporados en las campañas anteriores a 2002.

En esta campaña se rompe el bipartidismo tradicional y se abre la contienda electoral a candidaturas independientes. Este es el caso de Álvaro Uribe Vélez y su Movimiento Primero Colombia, quien se postuló por primera vez a la presidencia y obtuvo la mayor votación de la historia del país, rompiendo así con las viejas estructuras partidarias y abriendo paso a una organización electoral mucho más activa y planificada en lo estratégico.

Se evidencia que las campañas de los candidatos Álvaro Uribe Vélez y Horacio Serpa Uribe tuvieron un nivel de profesionalización medio-alto $\left(1 \leq I N P R O C O_{T}<1,5\right)$. Así mismo, se encontró que el nivel de profesionalización de las campañas en este periodo se explica por la dimensión comunicativa más que por la organizativa. En promedio, el nivel de profesionalización de la dimensión comunicativa es de 1,29, y el de la organizativa es de $1,15\left(\right.$ INPROCO $_{C}>$ INPROCO $\left._{O}\right)$.

\section{Medición y análisis del nivel de profesionalización total: campaña 2006}

La campaña de 2006 se caracterizó por ser una contienda electoral más corta debido a que se presentó, por primera vez en la historia reciente del país, la reelección inmediata del Presidente de la República, y la reforma 
en el sistema electoral que se derivó de este cambio implicó la realización de un referendo reeleccionista que retrasó el inicio de las candidaturas. En estas elecciones, el candidato/Presidente Álvaro Uribe Vélez tuvo un nivel de profesionalización medio-alto $\left(1 \leq I N P R O C O_{T}<1,5\right.$, mientras que Carlos Gaviria tuvo un nivel bajo $\left(0 \leq I_{N P R O C O}<0,5\right.$. Estos niveles extremos en el nivel de profesionalización de ambas candidaturas se pueden analizar desde el mismo fenómeno de la reelección y de la participación de un candidato de izquierda en la contienda.

De un lado, la campaña de Uribe empleó de manera intensiva los recursos y la infraestructura del Estado, y tuvo el apoyo de las clases políticas y los medios de comunicación. De otro, Gaviria representó un partido de izquierda y de oposición que se caracterizaba por sus fuertes principios ideológicos y disciplinas de partido que se apartan de la profesionalización de las campañas. De igual manera, se encontró que el nivel de profesionalización de las campañas en este periodo se explica por la dimensión comunicativa más que por la organizativa. En promedio, el nivel de profesionalización de la dimensión comunicativa es de 1,11, y de la organizativa es de 0,96 $\left(\right.$ INPROCO $_{C}>$ INPROCO $_{O}$.

\section{Medición y análisis del nivel de profesionalización total: campaña 2010}

En el proceso electoral de 2010 se evidencia que ambas campañas tuvieron un nivel alto de profesionalización en la dimensión comunicativa $\left(1,5 \leq I N P R O C O_{C}<2\right.$. No obstante, la campaña de Mockus tuvo una calificación superior a la de Santos, lo que se explica por la categoría discursiva, la cual en ambas campañas tiene un alto nivel de profesionalización $\left(1,5 \leq I N P R O C O_{C}\right.$ : Categoría discursiva $<2$, pero fue superior en la de Mockus debido a la incorporación intensiva de nuevas formas de discurso político.

Por su parte, tanto la categoría de procesos de comunicación como la de medios técnicos tienen un nivel alto de profesionalización ${ }^{2}$ y la misma calificación para ambas campañas, lo que se explica por la aparición de nuevas técnicas publicitarias como el storytelling y la campaña negativa, la preparación de los candidatos y de su grupo de trabajo para abordar los retos comunicativos, y la construcción de la expresión discursiva. Además, durante esta campaña se emplea de manera intensiva las nuevas tecnologías de la información y la política 2,0 (Plasser y Plasser, 2002.

En la campaña de 2010, la contienda electoral estaba representada por los candidatos Juan Manuel Santos, quien había sido el ministro más destacado del gobierno de turno, y por Antanas Mockus, reconocido por haber sido rector de la Universidad Nacional de Colombia y alcalde de la capital del país. En cuanto a la profesionalización de las campañas, se evidencia que la de Santos tuvo un nivel de profesionalización alto $\left(1,5 \leq I N P R O C O_{T}<2\right.$ mientras que la de Mockus tuvo un nivel medio-alto $(1 \leq$ INPROCO ${ }_{T}<1,5$. Asimismo, se encontró que el nivel de profesionalización de las campañas en este periodo se debe a la dimensión comunicativa más que a la organizativa. En promedio, el nivel de profesionalización de la dimensión comunicativa es de 1,82, y de la organizativa es de $1,31\left(\right.$ INPROCO $_{C}>$ INPROCO.

\section{Medición y análisis del nivel de profesionalización total: campaña 2014}

La contienda electoral de 2014 se caracterizó por incorporar nuevamente la campaña reeleccionista del Presidente Juan Manuel Santos, electo en la campaña de 2010. En estas elecciones, tanto el candidato/ Presidente Juan Manuel Santos, como su contender, Oscar Iván Zuluaga, tuvieron un nivel de profesionalización alto $\left(1,5 \leq I N P R O C O_{T}<2\right.$. Se encontró que el nivel de profesionalización de las campañas en este periodo se explica por la dimensión comunicativa más que por la organizativa. En promedio, el nivel de profesionalización de la dimensión comunicativa es de 1,86, y de la organizativa es de 1,69 $\left(\right.$ INPROCO $_{C}>$ INPROCO $_{O}$, aunque cabe resaltar que en la campaña de Santos se evidencia una paridad entre ambas dimensiones. 
Finalmente, en la figura 1 se evidencia el resultado final de la medición del nivel de profesionalización de las campañas presidenciales en Colombia durante los últimos 20 años, a partir de las dimensiones organizacional (INPROCOo) y comunicativa (INPROCOc), y del índice INPROCOT.

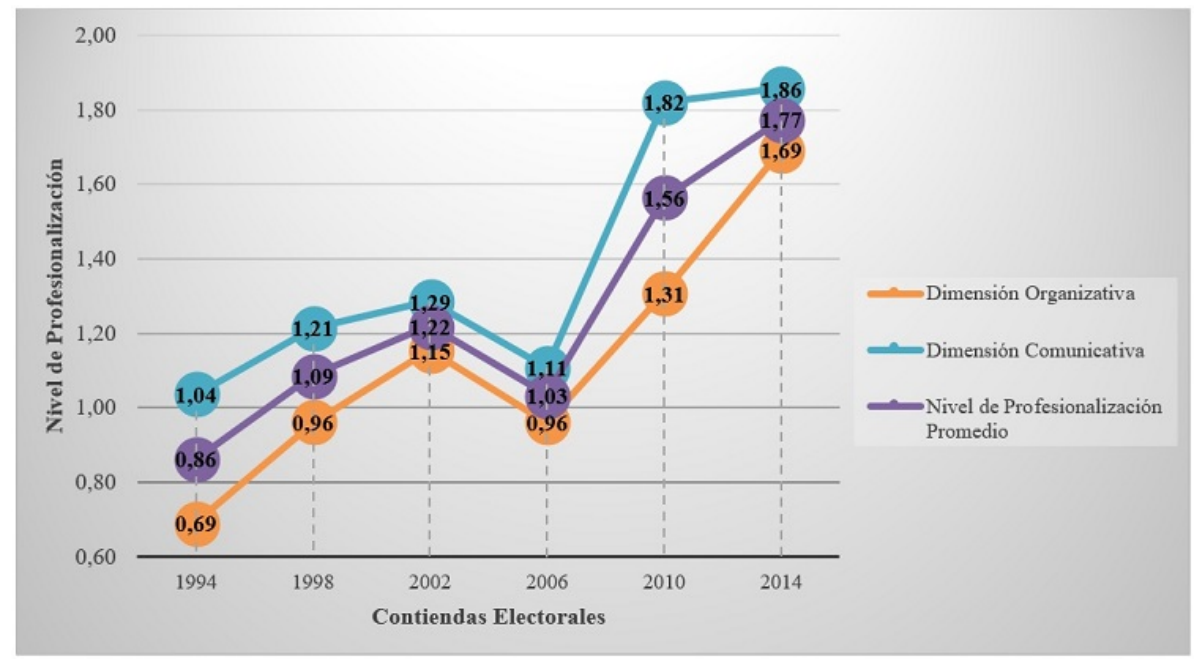

FIGURA 1.

Resultado de la medición y la evolución del nivel de profesionalización de las campañas presidenciales en Colombia 1994 - 2014

Fuente: elaboración propia a partir de Restrepo-Echavarría (2017)

\section{Conclusión}

Con esta investigación se logró evidenciar que en Colombia el nivel de profesionalización de las campañas se debe a la adaptación y perfeccionamiento de la dimensión comunicativa; aspecto que, en cada una de las campañas analizadas, aportó el mayor desarrollo y nivel de cubrimiento por las propuestas partidarias. Es decir, en contraste con la dimensión organizativa, la parte comunicacional fue la que mayor desarrollo profesional proporcionó en cada una de las contiendas electorales, tanto en las ganadoras como en las perdedoras.

De igual manera, se pudo inferir que la dimensión organizativa, que contó con menores índices de desarrollo, estuvo regida por las disposiciones partidarias del contexto electoral del país, que se modificaban de acuerdo a los sucesos coyunturales y al sistema electoral versátil de cada uno de los periodos analizados. Como evidencia de ello, determinados cambios en la normatividad electoral permitieron que colectividades y partidos modificaran sus estructuras organizacionales y se adaptaran con mayor facilidad a los nuevos retos estratégicos, lo que logró mantener una alta influencia en la forma como se desarrollaban las contiendas. En efecto, las candidaturas que provenían de la clase política, que tenían origen en los partidos tradicionales o que estaban apoyados por estas, evidenciaron mayor nivel de profesionalización en comparación con las campañas de candidaturas más jóvenes, como la de Antanas Mockus en la contienda electoral de 2010, o las de partidos políticos de centro izquierda, como el caso de Carlos Gaviria en el proceso electoral de 2006.

En ese sentido, aunque el nivel de profesionalización de las campañas ha venido presentando una evolución paulatina -al punto que en el 2014 se usaron de manera intensiva todas las herramientas de profesionalización de las campañas, tanto de la dimensión comunicativa como organizativa, pasando de un nivel de profesionalización medio $(0,86)$ en la campaña $1994\left(0,5 \leq \operatorname{INPROCO}_{T}<1\right)$ a un nivel alto $(1,77)$ en la de $2014\left(1,5 \leq I_{N P R O C O}<2\right)$ - las dinámicas políticas internas en el país han jugado un papel estructurante y determinante en el proceso de modernización, y ha quedado supeditado al candidato, su partido, y a variables económicas, políticas e ideológicas. 
A partir del análisis realizado comparativamente entre las campañas presidenciales que se ejecutaron en cada una de las décadas estudiadas (1990, 2000 y 2010) se logró concluir lo siguiente:

En las campañas presidenciales de 1994 y 1998 se logró evidenciar una tendencia creciente del nivel de profesionalización de las campañas. En efecto, el promedio de profesionalización de las campañas electorales pasó de un nivel medio (0,86 en la contienda electoral de 1994, a un nivel medio-alto (1,09 en la de 1998. Así mismo, se encontró que la dimensión comunicativa es la que explica en mayor medida el nivel de profesionalización de las campañas electorales durante la década de 1990, lo cual es consistente con los cambios en el sistema político del país en este periodo y con la coyuntura del momento. En concreto, durante ambas contiendas electorales, el nivel de profesionalización de la dimensión comunicativa tuvo un nivel medio-alto $\left(1 \leq \operatorname{INPROCO}_{C}<1,5\right.$.

En la década del 2000, se encontró que las campañas electorales de 2002 y 2006 tuvieron una tendencia decreciente del nivel de profesionalización. En efecto, aunque el promedio de profesionalización de las campañas electorales se mantuvo en un nivel medio-alto, se redujo de 1,22 en la contienda electoral de 2002, a 1,03 en la de 2006. Así mismo, se encontró que la dimensión comunicativa continúa explicando en mayor medida el nivel de profesionalización de las campañas electorales durante la década de 2000, lo cual es coherente con los cambios en el sistema electoral que generó la reelección y con el desarrollo de las nuevas tecnologías de la comunicación y la información.

En concreto, durante ambas contiendas electorales el nivel de profesionalización de la dimensión comunicativa tuvo un nivel medio-alto $\left(1 \leq I N P R O C O_{C}<1,5\right.$, mientras que la dimensión organizativa pasó de un nivel de profesionalización medio-alto en 2002 (1,15, a un nivel medio (0,96 en 2006, lo cual se explica por el bajo desempeño profesional de la campaña de Gaviria.

En la década de 2010 se evidencia una tendencia creciente del nivel de profesionalización de las campañas de 2010 y 2014, y una disminución en la brecha entre la dimensión organizativa y comunicativa. En efecto, el promedio de profesionalización de las campañas electorales se incrementó de un nivel medio-alto (1,56 en la campaña de 2010, a un nivel alto (1,77 en la de 2014, que se debe, además, a su alto nivel de profesionalización en la dimensión comunicativa y organizativa $\left(1,5 \leq \operatorname{INPROCO}_{C}<2\right.$ y $(1,5 \leq$ $I N P R O C O_{C}$ e INPROCO $O 2$ Lo anterior es consistente con el mayor esfuerzo de las campañas por modernizar su estructura e incorporar todas las técnicas profesionales de comunicación.

\section{Referencias}

Becassino, Á. (2008. La nueva política. El entorno y la comunicación electoral. Buenos Aires: Grijalbo.

Blumler, J. (1999. Tendencias de la comunicación política en las campañas electorales británicas. En A. Muñoz-Alonso y J. I. Rospir (eds, Democracia mediática y campañas electorales (pp. 111-124. Madrid, España: Ariel.

Castells, M. (2009. Comunicación y poder. Madrid: Alianza Editorial.

Crespo, I., Garrido, A., y Riorda, M. (2008. La conquista del poder. Elecciones y campañas presidenciales en América Latina. Buenos Aires: La Crujía.

Gibson, R., y Römmele, A. (2001. Changing Campaing Communication. A party-centered theory of professionalized campaigning. The Harvard International Journal of Press/Politics, 6(4, 31-43.

Gibson, R., y Römmele, A. (2009. Measuring the professionalization of political campaigning. Party Politics, 15(3, 265-293.

Kavanagh, D. (2004. Democracia partidaria y marketing político: ¿Sin lugar para aficionados? Diálogo politico, 21(1, $33-45$.

Maarek, P. (2009). Marketing Político y Comunicación. Clavespara una buena información política (2.a ed.. Barcelona: Paidós Ibérica.

Mancini, P. (1995). Americanización y modernización. Breve historia de la campaña electoral. En A. Muñoz-Alonso y J. I. Rospir (eds.), Comunicación política (pp. 141-168). Madrid, España: Editorial Universitas. 
Norris, P. (2001).¿Un círculo virtuoso? El impacto de las comunicaciones políticas en las democracias post-indstriales. Revista Española de Ciencia Política, 4, 7-33.

Norris, P. (2004) The evolution of election campaigns: Eroding political engagement? Recuperado de https://pdfs.sema nticscholar.org/6cef/9051c8815bfe29c590df15b4d6469d0ce11b.pdf

Orejuela, S. (2006). Proceso de Globalización a la profesionalización. Revista de Comunicación, 5, 55-87.

Plasser, F., y Plasser, G. (2002) La Campaña Global: Los nuevos gurúes del Marketing Político en acción. Buenos Aires: Temas.

Restrepo-Echavarría, N. J. (2015) La profesionalización de las campañas electorales: las elecciones presidenciales de Colombia 2010. Revista Española de Ciencia Política, 38, 85-114.

Restrepo-Echavarría, N. J. (2017) La profesionalización de las campañas electorales en Colombia: las elecciones presidenciales en Colombia 1994-2014 (tesis doctoral). Universidad Complutense de Madrid, Madrid, España.

Restrepo-Echavarría, N. J. (2018) La influencia de los medios de comunicación en las campañas electorales en Colombia. Sociedad civil y partidos políticos. Revista Dialogo Politico, 34(1), 50-62.

Restrepo-Echavarría, N. J., Rodríguez-Díaz, R., y Castromil, R. (2018). Proposal of an indicator to measure the professionaliza on of elec on campaigns: The case of Colombia. El profesional de la información, 27(2), 289-299.

Rocha, F. (2007). La profesionalización de las campañas electorales en Brasil (1989-2006). Salamanca: Universidad de Salamanca.

Rospir, J. (1999). La globalización de las campañas electorales. En A. Muñoz-Alonso y J. Rospir (eds.), Democracia mediática y campañas electorales (pp. 55-88). Madrid, España: Ariel.

Swanson, D., y Mancini, P. (1996). Politics, Media and Modern Democracy: An International Study of Innovations in Electoral Campaigning and their Consequences. Washington: Praeger.

\section{Notas}

* Artículo de investigación

$11 \leq I^{2} P R O C O_{C}$ : Categoría Dicursiva,procesos de comunicación y medios técnicos $<1,5$

$21,5 \leq$ INPROCO $_{C}$ : Categoría procesos de comunicación y medios técnicos $<2$

Licencia Creative Commons CC BY 4.0

Cómo citar este artículo: Restrepo-Echavarría, N. J., y Gómez-Restrepo, A. M. (2019). Las estrategias de profesionalización de las campañas presidenciales en Colombia desde 1994-2014. Signo y Pensamiento, 38(74). https://doi.org/10.11144/Javeriana.syp38-74.epcp 